PROCEEDINGS OF THE AMERICAN MATHEMATICAL SOCIETY

Volume 124, Number 9, September 1996

\title{
CONSTANT-TO-ONE EXTENSIONS OF SHIFTS OF FINITE TYPE
}

\author{
DORIS FIEBIG
}

(Communicated by Mary Rees)

\begin{abstract}
Any transitive shift of finite type has a transitive constant-to-one extension which is not of finite type.
\end{abstract}

Searching for an analog of Rudolph's result in ergodic theory ("any weakly mixing constant-to-1 extension of a Bernoulli system is also Bernoulli" [R2]), F. Blanchard posed the question of whether any transitive constant-to-1 extension of a shift of finite type is also of finite type [B]. So far, such extensions have been shown to be coded $[\mathrm{B}]$, and to be of finite type if one assumes the extension to be sofic [BH]. We answer the original question and show that any shift of finite type has a transitive constant-to-1 extension which is not of finite type (and thus not even sofic).

We introduce some notation and recall some definitions. Let $A$ be a finite set. A subshift $S$ is a closed shift invariant subset of the compact set $A^{\mathbf{Z}}$ together with the (left) shift map restricted to $S$. For a point $s=\left(s_{i}\right)_{i \in \mathbf{Z}} \in S$ let $s[n, m]$ denote the subblock $s_{n} s_{n+1} \cdots s_{m}$ of $s(-\infty \leq n \leq m \leq \infty)$. An $S$-block is a finite subblock of some point in $S$. The set of elements in $A$ which are $S$-blocks is the symbol set of $S$. A subshift $S$ is transitive if for any pair of $S$-blocks, say $a$ and $c$, there is an $S$-block $a b c$ connecting them. A shift of finite type is a subshift $S$ which is defined by excluding a finite set of blocks, say $B$, that is, a point $s \in A^{\mathbf{Z}}$ belongs to $S$ if and only if no subblock of $s$ is an element of $B$.

Let $S$ and $T$ be subshifts. A factor map $\pi: S \rightarrow T$ is a shift commuting continuous map from $S$ onto $T$. The subshift $S$ is then said to be an extension of $T$. The map $\pi$ is constant-to- 1 if all fibers $\pi^{-1}(t), t \in T$, have the same finite cardinality.

Theorem. Let $T$ be an infinite transitive shift of finite type. Let $d \geq 2$ be an integer. Then there is a transitive subshift $S$ which is not a shift of finite type and a factor map $\pi: S \rightarrow T$ such that all fibers have cardinality $d$.

Proof. We need some preparations. By recoding we may assume that $T$ is given by a finite excluding block system where all blocks have length 2 [DGS]. Then a block is a $T$-block if and only if all its subblocks of length 2 are $T$-blocks. Let $\Sigma$ be the symbol set of $T$. Fix a shortest $T$-block $b_{0} \cdots b_{n}$ with $n \geq 1$ and $b_{0}=b_{n}$. Since $T$ is not finite, there is some $i, 1 \leq i \leq n$, and a symbol $\sigma \in \Sigma$ with $\sigma \neq b_{i-1}$ and for which $\sigma b_{i}$ is a $T$-block. We may assume that $i=1$ (if necessary replace $b_{0} \cdots b_{n}$ by $\left.b_{i-1} \cdots b_{n} b_{1} \cdots b_{i}\right)$. Fix a shortest $T$-block $a_{0} \cdots a_{k}$ with $k \geq 1, a_{0}=b_{n}$

Received by the editors January 6, 1995 and, in revised form, March 14, 1995.

1991 Mathematics Subject Classification. Primary 58F03, 54H20; Secondary 58F08, 28D05.

(C)1996 American Mathematical Society 
and $a_{k}=\sigma$. Let $A=a_{1} \cdots a_{k}$ and $B=b_{1} b_{2} \cdots b_{n} . A$ and $B$ satisfy the following properties:

-Any finite concatenation of the blocks $A B$ and $B$ is a $T$-block.

$-\sigma$ does not occur in $B$ and $b_{n}$ does not occur in $A$, thus $A$ and $B$ cannot overlap.

-Let $t \in T$. If for some $-|B|<i \leq 0$ it holds that $t[i, i+|B|-1]=B$, then the index $i$ is unique. The analogue holds for the block $A$.

Let $\Sigma^{\prime}$ be a copy of $\Sigma$ and fix a bijection $f: \Sigma \rightarrow \Sigma^{\prime}$. We extend $f$ to a map on blocks with all symbols in $\Sigma$, which we call again $f$, by $f\left(u_{1} \cdots u_{n}\right):=$ $f\left(u_{1}\right) \cdots f\left(u_{n}\right)$.

Let $C, D, E$ be $T$-blocks, $t \in T$ and $n \in \mathbf{Z}$. We say $t_{n}$ lies in the block $D$ if for some $n-|D|<i \leq n$ it holds that $t[i, i+|D|-1]=D$. We say $t_{n}$ lies in $D$ within the context $(C, E)$ if there is some $n-|D|<i \leq n$ such that $t(-\infty, i-1]$ ends with $C$ and $t[i, \infty)$ begins with $D E$. Thus, if $t_{n}$ lies in $D$ within the context $(C, E)$, then $t_{n}$ lies in $C D E$.

Observe that the notion " $t_{n}$ lies in $D$ " does not only mean that the $n$th symbol of $t$ is a subblock of $D$ but also that $D$ is a subblock of $t[n-|D|+1, n+|D|-1]$. The same applies to the notion of " $t_{n}$ lies in $D$ within the context $(C, E)$ ".

For each $k \geq 1$ let $L(k):=A B^{k}$ and $R(k)=B^{k} A$. We define a shift commuting (non-continuous) map $\Phi: T \rightarrow\left(\Sigma^{\prime} \cup \Sigma\right)^{\mathbf{Z}}$ by defining $\Phi(t)_{0}$ for each $t \in T$. Let $t \in T$.

Case 1: $t_{0}$ lies in $B$. If $t_{0}$ lies in $B^{n}$ within the context $(L(n) L(n-1) \cdots L(1) A$, $A R(1) R(2) \cdots R(n))$ for some $n \geq 100$, then let $\Phi(t)_{0}=f\left(t_{0}\right)$ and let $\Phi(t)_{0}=t_{0}$ otherwise.

Case 2: If $t_{0}$ lies in $A$ and, for some $n \geq 100, t_{0}$ lies in $L(n-2) L(n-3)$ $\cdots L(2) L(1) A$ within the context $\left(L(n) L(n-1), B^{n}\right)$, then let $\Phi(t)_{0}=t_{0}$.

Case 3: If $t_{0}$ lies in $A$ and, for some $n \geq 100, t_{0}$ lies in $A R(1) R(2) \cdots$ $R(n-3) R(n-2)$ within the context $\left(B^{n}, R(n-1) R(n)\right)$, then let $\Phi(t)_{0}=t_{0}$.

Case 4: None of the above cases apply. Then let $\Phi(t)_{0}=f\left(t_{0}\right)$.

By the non-overlapping properties of the blocks $A$ and $B$ the map $\Phi$ is well defined.

Fix $d-1$ copies of $\Sigma$, say $\Sigma^{(i)}, 1 \leq i<d$, and let $\Sigma^{(0)}=\Sigma$. For each $1 \leq i<d$ fix a bijection from $\Sigma$ to $\Sigma^{(i)}$, and denote the image of $u \in \Sigma$ by $u^{(i)}$. For $u \in \Sigma$ we also write $u^{(0)}$. We consider the symbols $f(u) \in \Sigma^{\prime}(u \in \Sigma)$ appearing in points in the image of $\Phi$ as variables where $f(u)$ can be replaced by any of the $u^{(i)}, 0 \leq i<d$, with the convention that $u^{(0)}=u$. We do these replacements according to the following rules. (Recall that $\sigma \in \Sigma$ is the last symbol in the block $A$ and that $\sigma$ does not occur in $B$.)

Let $x w y$ be a block appearing in some point of $\Phi(T)$, where $x, y \in \Sigma^{\prime}$ and $w$ is either the empty block or consists only of symbols in $\Sigma$. Then $x=f(u)$ and $y=f(v)$ for unique $u, v \in \Sigma$. The replacement $u^{(i)} w v^{(j)}$ is allowed if and only if $(u \neq \sigma$ and $i=j)$ or $(u=\sigma$ and $j=i+1 \bmod d)$.

Now we replace all those $f(u) \in \Sigma^{\prime}$ in points of $\Phi(T)$ according to the described rule. Observe that we get from each point $y \in \Phi(T)$, which sees a symbol from $\Sigma^{\prime}$, exactly $d$ points in $\left(\Sigma^{(0)} \cup \cdots \cup \Sigma^{(d-1)}\right)^{\mathbf{Z}}$. A point $y \in \Phi(T)$, which does not see a symbol from $\Sigma^{\prime}$, will be fixed by the replacements. Thus we have assigned to each point $t \in T$ a set $\widehat{\Phi(t)} \subset\left(\Sigma^{(0)} \cup \cdots \cup \Sigma^{(d-1)}\right)^{\mathbf{Z}}$ of cardinality either $d$ or 1 . Let

$$
\widehat{\Phi(T)}=\left\{x \in\left(\Sigma^{(0)} \cup \cdots \cup \Sigma^{(d-1)}\right)^{\mathbf{Z}} \mid x \in \widehat{\Phi(t)} \text { for some } t \in T\right\} \text {. }
$$


Let $S$ be the closure of $\widehat{\Phi(T)}$ in $\left(\Sigma^{(0)} \cup \cdots \cup \Sigma^{(d-1)}\right)^{\mathbf{Z}}$. Let $\pi: S \rightarrow T$ be the 1-block map which erases the superscripts, i.e. $\pi(s)_{0}=u$ if $s_{0}=u^{(i)} \in \Sigma^{(i)}$ for some $i$ (recall that $u^{(0)}=u$ ).

We will see that $\Phi: T \rightarrow\left(\Sigma^{\prime} \cup \Sigma\right)^{\mathbf{Z}}$ is almost continuous (Claim $\left.1+2\right), \pi$ is a factor map from $S$ onto $T$ (Claim 3), $\pi$ is constantly $d$-to-1 (Claim 5), and $S$ is transitive (Claim 6) and not a shift of finite type (Claim 7). Recall that $L(k)=A B^{k}$ and $R(k)=B^{k} A, k \geq 1$.

Claim 1. Let $t \in T$ such that $t_{0}$ lies in $A B^{k} A$ for some $k \geq 1$. Then $\Phi(t)_{0}$ is determined by $t[-n, n]$ for some $n=n(k)$.

Proof. If $t_{0}$ lies in $B^{k}$, then Case 1 applies and $\Phi(t)_{0}$ is determined by $t[-n, n]$, for $n=|L(k) \cdots L(2) L(1) A|+\left|B^{k}\right|$. If $t_{0}$ lies in the first $A$, then Case 2 applies if $t_{0}$ lies in $L(K-2) L(K-3) \cdots L(2) L(1) A$ within the context $\left(L(K) L(K-1), B^{K}\right)$, where $K=\max (100, k+2)$ and Case 3 applies if $t_{0}$ lies in $A R(1) R(2) \cdots R(K-3) R(K-2)$ within the context $\left(B^{K}, R(K-1) R(K)\right)$, where $K=\max (100, k+1)$. If neither Case 2 nor Case 3, then Case 4 applies. Thus $\Phi(t)_{0}$ is determined by $t[-n, n]$, for $n=\left|B^{K} A R(1) R(2) \cdots R(K-1) R(K)\right|$, where $K=\max (100, k+2)$. The case that $t_{0}$ lies in the second $A$ is symmetric.

We specify four $T$-orbits. Let $B^{\infty}$ denote the periodic $T$-orbit obtained from bi-infinite concatenations of $B$. Let $B^{\infty} A B^{\infty}$ denote the orbit of the point $t$ with $t(-\infty,-1]=\cdots B B B$ and $t[0, \infty)=A B B B \cdots$. Let $B^{\infty} A R(1) R(2) R(3) \cdots$ denote the orbit of $t$ with $t(-\infty,-1]=\cdots B B B$ and $t[0, \infty)=A R(1) R(2) R(3) \cdots$, similarly $\cdots L(3) L(2) L(1) A B^{\infty}$.

Claim 2. Let

$$
D=T-\left\{B^{\infty}, B^{\infty} A B^{\infty}, B^{\infty} A R(1) R(2) R(3) \cdots, \cdots L(3) L(2) L(1) A B^{\infty}\right\} .
$$

Then for all $t \in D$ and all $k \geq 0$ there is some $n=n(t, k) \geq 0$ such that for all $t^{\prime} \in T$ with $t^{\prime}[-n, n]=t[-n, n]$ we have $\Phi\left(t^{\prime}\right)[-k, k]=\Phi(t)[-k, k]$.

Proof. Since $D$ is shift invariant it suffices to prove the claim for $k=0$. Let $R$ be the shift of finite type in $T$ in which all points are bi-infinite concatenations of the blocks $A B$ and $B$. Then $T-R$ is a subset of $D$. Let $t \in T-R$. Then there is $m \geq 0$ such that $t[-m, m]$ is not an $R$-block. Thus, if $t_{0}$ satisfies Case 1 , Case 2 or Case 3, then for some $n$ with $n<m$. Therefore $\Phi(t)_{0}$ is determined by $t[-n, n]$ for large enough $n$. Now let $t \in R \cap D$. It remains to consider the case where Claim 1 does not apply. Then if $t_{0}$ lies in $A$ we have that $t$ lies in $B^{\infty} A B^{\infty}$, a contradiction. Thus $t_{0}$ lies in $B$. Since Claim 1 does not apply, $t_{n}$ lies in $B$ for all $n \leq 0$ or for all $n \geq 0$. Assume that $t_{n}$ lies in $B$ for all $n \leq 0$ (the other case is similar). Let $p$ be the least integer for which $t_{p}$ lies in $A$. Since $t$ is not in the orbit $B^{\infty} A R(1) R(2) R(3) \cdots$, there is some $N$ such that $t_{p}$ does not lie in $A$ within the context $\left(B^{N}, R(1) R(2) \cdots R(N)\right)$. Then, $\Phi(t)_{0}$ is determined by $t[-L, p+L]$, where $L=|A R(1) R(2) \cdots R(N)|$.

Claim 3. Let $s \in \widehat{\Phi(t)}$ for some $t \in T$. Then $\pi(s)=t$. Thus, since $\widehat{\Phi(T)}$ is dense in $S$ and $\pi$ is continuous, $\pi: S \rightarrow T$ is an onto factor map.

Proof. $s$ is obtained from $\Phi(t)$ by replacing the symbols $f(u) \in \Sigma^{\prime}$ by some of the $u^{(i)}$ and leaving symbols from $\Sigma$ fixed. $s_{n} \in\left\{u^{(i)}, 0 \leq i<d\right\}$ only if $t_{n}=u$. Thus $\pi(s)=t$. 
Claim 4. Let $t \in D$. Then $\Phi(t)$ sees a symbol from $\Sigma^{\prime}$.

Proof. If $t$ never sees the block $L(100) L(99) \cdots L(2) L(1) A B^{100}$ and never sees the block $B^{100} A R(1) R(2) \cdots R(99) R(100)$, then $\Phi(t)_{n}=f\left(t_{n}\right) \in \Sigma^{\prime}$ for some $n$. In fact, since $t$ does not lie in the orbit $B^{\infty}$ and the block $B$ cannot overlap itself, there is some $n$ such that $t_{n}$ does not lie in $B$. Therefore, Case 4 applies for $t_{n}$. Now assume that, for some $n, t_{n}$ lies in $L(99) \cdots L(2) L(1) A$ within the context $\left(L(100), B^{100}\right)$ (the other case is similar). Since $t$ is not in the orbit $\cdots L(3) L(2) L(1) A B^{\infty}$, there is a maximal $k \geq 100$ such that $t_{n}$ lies in $L(99) \cdots L(2) L(1) A$ within the context $\left(L(k) L(k-1) \cdots L(100), B^{k}\right)$. Let $m \leq n$ be maximal such that $t_{m}$ lies in $A$ within the context $\left(L(k), B^{k-1} A\right)$. By the maximality of $k$, Case 4 applies for $t_{m}$ and thus $\Phi(t)_{m}=f\left(t_{m}\right) \in \Sigma^{\prime}$.

Claim 5. $\pi: S \rightarrow T$ is constantly d-to-1.

Proof. First we show that any point in $T$ has at most $d$ preimages under $\pi$. Assume there is a point $t \in T$ with at least $d+1$ preimages, say $s^{(1)}, s^{(2)}, \ldots, s^{(d+1)}$. Let $k \geq 0$ be so large that $s^{(i)}[-k, k], 1 \leq i \leq d+1$, are pairwise distinct blocks. Then for $n \geq 0$ and $1 \leq i \leq d+1$ there is $t^{(i)}=t^{(i, n)} \in T$ such that there is $y^{(i)}=y^{(i, n)} \in \widehat{\Phi\left(t^{(i)}\right)}$ with $y^{(i)}[-n, n]=s^{(i)}[-n, n]$. By Claim 3 it holds that $\pi\left(y^{(i)}\right)=t^{(i)}$ and thus $t^{(i)}[-n, n]=\pi\left(s^{(i)}\right)[-n, n]=t[-n, n]$. Now we consider different possibilities for $t$ and choose in each case a suitable $n$ to get a contradiction.

Assume first that $t \in D$. Choose $n=n(t, k)$ from Claim 2. Then

$$
\Phi\left(t^{(1)}\right)[-k, k]=\Phi\left(t^{(2)}\right)[-k, k]=\cdots=\Phi\left(t^{(d+1)}\right)[-k, k] .
$$

Thus, by the replacement rules, $\operatorname{card}\left\{y^{(i)}[-k, k] \mid 1 \leq i \leq d+1\right\} \leq d$, contradicting the distinctness of $s^{(i)}[-k, k], 1 \leq i \leq d+1$.

If $t$ lies in the orbit $B^{\infty}$, then if $s$ and $s^{\prime}$ are preimages of $t$ and for some $0 \leq i<d$ we have $s_{0} \in \Sigma^{(i)}$ and $s_{0}^{\prime} \in \Sigma^{(i)}$, then $s_{0}=s_{0}^{\prime}$. Thus, for some $s=s^{(m)}$ it holds that $s[p, p+1]=u^{(i)} v^{(j)}$ for some $p$, and some $0 \leq i, j<d$ with $i \neq j$. Let $n=|p+1|+|B|$ and $t^{\prime}=t^{(m)}$. Then Case 1 coding applies to $t_{p}^{\prime}$ and $t_{p+1}^{\prime}$; thus $\Phi\left(t^{\prime}\right)[p, p+1]=f(u) f(v)$ or $\Phi\left(t^{\prime}\right)[p, p+1]=u v$. Since $s[p, p+1]=u^{(i)} v^{(j)}$ with $i \neq j$, we have $\Phi\left(t^{\prime}\right)[p, p+1]=f(u) f(v)$. Thus by the replacement rules, $u=\sigma$, a contradiction, since $u$ is a symbol of $B$.

If $t(-\infty,-1]=B^{\infty}$ and $t[0, \infty)=A B^{\infty}$, let $n=k+|A B B|$. Then $\Phi\left(t^{(i)}\right)[-k, k]$ $=t[-k, k]$ or $\Phi\left(t^{(i)}\right)[-k, k]=t[-k,-1] f(A) t[|A|, k]$, for each $1 \leq i \leq d+1$. Thus, since $A$ sees $\sigma$ only as its last symbol, $\operatorname{card}\left\{y^{(i)}[-k, k] \mid 1 \leq i \leq d+1\right\} \leq d$, a contradiction.

$$
\begin{aligned}
& \text { If } t(-\infty,-1]=B^{\infty} \text { and } t[0, \infty)=A R(1) R(2) \cdots, \text { w.l.o.g. } \\
& \qquad k=|A R(1) R(2) \cdots R(m)|-1 \quad \text { for some } m \geq 2 .
\end{aligned}
$$

Let $n=k+n(1)+\cdots+n(m)$, where the $n(i)$ are from Claim 1. Then $\Phi\left(t^{(1)}\right)[0, k]=$ $\Phi\left(t^{(2)}\right)[0, k]=\cdots=\Phi\left(t^{(d+1)}\right)[0, k]=\Phi(t)[0, k]=t[0, k]$. Since $B$ does not see the symbol $\sigma$, the replacement rules imply $\operatorname{card}\left\{y^{(i)}[-k, k] \mid 1 \leq i \leq d+1\right\} \leq d$, a contradiction.

The case that $t$ is in the orbit $\cdots L(2) L(1) A B^{\infty}$ is symmetric to the last case. This proves that $\pi$ is at most $d$-to- 1 . We now show that any point has at least $d$ preimages.

First let $t \in D$. By Claim $4 \Phi(t)$ sees a symbol from $\Sigma^{\prime}$. Thus $\widehat{\Phi(t)}$ has cardinality $d$. All points of $\widehat{\Phi(t)}$ belong to $S$ by definition. 
If $t$ lies in the orbit $B^{\infty} A B^{\infty}$, then $\Phi(t)$ lies in the orbit $B^{\infty} f(A) B^{\infty}$ (Case 1 and Case 4). By the replacement rules $t$ has $d$ preimages.

For every $n \geq 100$ there is a point $u \in T$ such that $u_{0}$ lies in $B^{n}$ within the context $(L(n) L(n-1) \cdots L(2) L(1) A, A R(1) R(2) \cdots R(n-1) R(n))$. Applying Cases 1-3 shows that $\Phi(u)_{0}$ lies in $f(B)^{n}$ within the context $(L(n-2) L(n-3) \cdots L(2) L(1) A$, $A R(1) R(2) \cdots R(n-3) R(n-2))$. Since $B$ does not see the symbol $\sigma$, the replacement rule shows that for each $0 \leq i<d$ there is an $S$-block $L(n-2) L(n-3)$ $\cdots L(2) L(1) A\left(B^{(i)}\right)^{n} A R(1) R(2) \cdots R(n-3) R(n-2)$, where $B^{(i)}$ has all symbols in $\Sigma^{(i)}$ and is mapped to $B$. By compactness of $S$ this proves that any point in one of the orbits $B^{\infty}, B^{\infty} A R(1) R(2) \cdots$ and $\cdots L(2) L(1) A B^{\infty}$ has at least $d$ preimages.

Claim 6. $S$ is transitive.

Proof. The last argument used in the proof of Claim 5 actually proved that points in the orbits $B^{\infty}, B^{\infty} A R(1) R(2) \cdots$ and $\cdots L(2) L(1) A B^{\infty}$ can be approximated arbitrarily well by points from $\left\{x \in\left(\Sigma^{(0)} \cup \cdots \cup \Sigma^{(d-1)}\right)^{\mathbf{Z}} \mid x \in \widehat{\Phi(t)}\right.$ for some $t \in D\}$. An analogous argument shows that there are $S$-points which are mapped to the orbits $\left(B^{n} A\right)^{\infty}, n \geq 1$, accumulating at $B^{\infty} A B^{\infty}$. Thus $S$ is the closure of $\left\{x \in\left(\Sigma^{(0)} \cup \cdots \cup \Sigma^{(d-1)}\right)^{\mathbf{Z}} \mid x \in \widehat{\Phi(t)}\right.$ for some $\left.t \in D\right\}$. Now let $a$ and $b$ be two $S$-blocks. Choose $t \in D$ such that there is $y \in \widehat{\Phi(t)}$ with $y[1,|a|]=a$. By Claim 2 we can choose $p$ so large that $t^{\prime} \in T, t[-p, p]=t^{\prime}[-p, p]$ implies $\Phi(t)[1,|a|]=\Phi\left(t^{\prime}\right)[1,|a|]$. Now choose a point $u \in D$ such that $u[-p, p]=t[-p, p]$, $u[N, \infty)=A B A B A B \cdots$ and $u(-\infty,-M]=\cdots A B A B A B$ for some $M, N>0$. By Claim 2 we can choose $m, n \geq 0$ so large that $u^{\prime} \in T, u^{\prime}[-m, n]=u[-m, n]$, then $\Phi\left(u^{\prime}\right)[-M, N]=\Phi(u)[-M, N]$. We may assume that $u[-m, n]$ begins with $A$ and ends with $B$. Let $u^{(a)}=u, m^{(a)}=m$ and $n^{(a)}=n$. Do the same for the block $b$ to obtain $u^{(b)}, m^{(b)}$ and $n^{(b)}$. Then or every $p$, there is a point $x^{(p)} \in T$ with $x^{(p)}(-\infty,-1]=u^{(a)}\left(-\infty, n^{(a)}\right]$ and $x[0, \infty)=(A B)^{p} u^{(b)}\left[-m^{(b)}, \infty\right)$. We have by the choice of $u^{(a)}, u^{(b)}, n^{(a)}$ and $m^{(b)}$ that $\Phi\left(x^{(p)}\right)(-\infty,-1]=\Phi\left(u^{(a)}\right)\left(-\infty, n^{(a)}\right]$ and $\Phi\left(x^{(p)}\right)[0, \infty)=(f(A) B)^{p} \Phi\left(u^{(b)}\right)\left[-m^{(b)}, \infty\right)$. By the definition of $u^{(a)}$ and $u^{(b)}$ and since $A$ sees the symbol $\sigma$ exactly once, there is a $p$ such that one of the points in $\widehat{\Phi\left(x^{(p)}\right)}$ sees first the block $a$ and after a while the block $b$.

Claim 7. $S$ is not a shift of finite type.

Proof. Let $y^{(1)}$ be the unique preimage of $B^{\infty}$ with symbols in $\Sigma^{(1)}$, and let $\pi\left(y^{(1)}\right)[0,|B|-1]=B$. Let $B^{(1)}=y^{(1)}[0,|B|-1]$. If $S$ were a shift of finite type, then there would be an orbit of the form $\left(B^{(1)}\right)^{\infty} w\left(B^{(1)}\right)^{\infty}$ which is not the orbit of $\left(B^{(1)}\right)^{\infty}$. Choose $s$ from this orbit with $s(-\infty,-1]=\left(B^{(1)}\right)^{\infty}$ and with the property that $s[0, \infty)$ does not begin with $B^{(1)}$. Choose $n \geq 2$ so large that $|A R(1) R(2) \cdots R(n-1)| \geq|w|$. Then choose $m$ so large that $m|B| \geq$ $|A R(1) R(2) \cdots R(n)|$. There is some $t \in T$ such that $\Phi(t)[-n|B|,|w|+m|B|]=$ $f(B)^{n} u f(B)^{m}$, where $|u|=|w|$. Thus, $t[0, \infty)$ begins with $A R(1) R(2) \cdots R(n)$ and $t[|w|, \infty)$ begins with $B^{m}$. By the choice of $n$ and $m$ this implies that $A$ is a subblock of $B^{m}$, a contradiction.

Remark. $S$ is a synchronized system but does not have specification. We think that non-synchronized constant-to- 1 extensions can be obtained by a more elaborate variant of the above construction. 


\section{REFERENCES}

[B] F. Blanchard, Extensions à fibre constante, Ergodic Theory Dynamical Systems, 11, (1991), 7-17. MR 92f:58052

[BH] F. Blanchard and G. Hansel, Sofic constant-to-one extensions of subshifts of finite type, Proc. Amer. Math. Soc., 112, (1991), 259-265. MR 91m:54050

[DGS] M. Denker, C. Grillenberger, and K. Sigmund, Ergodic theory on compact spaces, Lecture Notes in Math., vol. 527, Springer, New York, 1976, MR 56:15879

[R1] D. Rudolph, If a two-point extension of a Bernoulli shift has an ergodic square then it is Bernoulli, Israel J. Math., 30, (1978), 159-180. MR 80h:28028a

[R2] - If a finite extension of a Bernoulli shift has no finite rotation factors, it is Bernoulli, Israel J. Math. 30 (1978), 193-206. MR 80h:28028b

Institut für Angewandte Mathematik, Universität Heidelberg, Im Neuenheimer Feld 294, 69120 Heidelberg, Germany

E-mail address: fiebig@math.uni-heidelberg.de 